\title{
A projection method for motion of triple junctions by level sets
}

\author{
KURT A. SMITH ${ }^{\dagger}$ \\ Department of Chemical Engineering, Northwestern University, Evanston, Illinois 60208, USA \\ FRANCISCO J. SOLIS \\ Department of Physics and Astronomy, Arizona State University, Tempe, Arizona 85287, USA \\ AND \\ DAVID L. CHOPP \\ Department of Engineering Science and Applied Mathematics, Northwestern University, \\ Evanston, Illinois 60208, USA
}

[Received 5 September 2001 and in revised form 4 March 2002]

\begin{abstract}
We develop a projection method to treat the motion of multiple junctions (such as contact lines) in the level set formulation. Multiple junctions are relevant to many fields including fluid dynamics, foams, and semiconductor manufacture. In the level set method an interface is defined as the zero level set of a smooth function. For an $N$-phase system the location of all interfaces can be specified by $N-1$ functions (hence only one level set function is needed for a two-phase system). For $N>2$ we describe a symmetric projection of the $N$ level set functions onto an $N-1$ dimensional manifold. This reduction in phase space eliminates unacceptable values of the level set functions (such as cases where more than one is positive at a given point.) This prevents the formation of vacuums or overlaps at multiple junctions during interface evolution. Further, this method can be applied to any number of phases and spatial dimensions. We present two- and three-dimensional results showing that the method gives correct equilibrium contact angles and produces accurate dynamics in multi-phase fluids.
\end{abstract}

Keywords: multiple junctions; algorithms; contact line motion; multiphase flow.

\section{Introduction}

The level set method as proposed by Osher and Sethian [8] is a novel method for capturing the evolution of complex interfaces. In this method, the interface is represented as the zero level surface of a 'level set function' of one higher dimension. The evolution of the surface is then embedded in an evolution equation for the level set function itself which is solved on a fixed Eulerian mesh. The method has many well established advantages over competing front tracking algorithms. These include a natural handling of topological changes in the interface, easy extension to any number of spatial dimensions, and a fundamental connection to numerical methods for hyperbolic conservation laws which enables the stable and accurate evolution of corners and cusps in the interface.

However, the choice of representing the surface implicitly also restricts the types of surfaces which can be represented, namely orientable manifolds. It also effectively excludes the construction of surfaces which have triple junctions such as can arise in multi-phase fluid problems. For example, the spreading of a liquid droplet on a solid surface involves air, liquid, and solid phases.

† Corresponding author. Email: k-smith5@northwestern.edu 
Attempts at extending the level set method to include more than two phases have been mostly successful, but they suffered from some critical drawbacks. In [7], each phase is represented by a separate level set function. The difficulty with this approach is to establish an appropriate constraint condition at a triple junction that couples the three functions at a single point. Note that this is not an issue when only two phases are present because the two phases are implicitly coupled through the use of a single level set function. In [7], this coupling is enforced by a type of interface surgery performed every time step. In this approach, the different phase interfaces move independently according to the given speed law. After the interface has moved, voids and overlaps are corrected by replacing each level set function $\phi_{i}$ by $\frac{1}{2} \max _{j \neq i}\left(\phi_{i}-\phi_{j}\right)$. This approach is an ad hoc solution which has no physical basis, but has been shown to produce reasonable results for some problems.

In [14], a variational formulation is used to remove the overlaps/voids through an additional constraint equation. While this approach does effectively eliminate overlaps and voids, its application is restricted to problems at near-equilibrium conditions (see also [15]). More general dynamic interface motion is not addressed. It also depends heavily on the use of reinitialization instead of using velocity extensions. It has been shown in [6] that the repeated use of reinitialization can lead to substantial errors in volume conservation, though these errors can be reduced using more sophisticated reinitialization techniques.

In [4], the authors represent the multiple interfaces by using a binary approach. For $N$ phases, $\left\lceil\log _{2} N\right\rceil$ level set functions are used. Phase $i$ is located by taking a binary representation for $i$, call it $d_{1} d_{2} \ldots d_{n}$, and then

$$
\{x \text { in Phase } i\}=\left\{x: \phi_{j}(x)\left\{\begin{array}{ll}
>0 & \text { if } d_{j}=1 \\
<0 & \text { if } d_{j}=0
\end{array}\right\}\right\}
$$

This representation effectively eliminates voids and overlaps when the number of phases is $N=2^{m}$. However, when $N \neq 2^{m}$ for some integer $m$, voids can appear in the form of non-existent phases. For example, if $N=3$, then two level set functions are used to represent the three phases labelled 0 , 1,2 . A fourth phase corresponding to $\phi_{1}, \phi_{2}>0$ is unaccounted for among the physical phases in the application, but can appear numerically. However, it should be pointed out that for the applications in image processing to which this method has been employed it has worked well.

The real issue here is that the space of permissible configurations for $N$ level set functions at a point is $N-1$ dimensional. The approaches discussed above use $N$ or $\left\lceil\log _{2} N\right\rceil \operatorname{dimensions}$. In this paper, we build an $(N-1)$-dimensional representation which correctly handles the triple junction in such a way that overlaps and voids cannot appear. This is accomplished through the use of projections of the level set functions onto a subspace in which there is a one-to-one map between points in the subspace and allowable values of the level set functions. The evolution of the interfaces is done through a combination of steps in both the projected and real spaces. The end result is a numerical method which can generalize to any number of phases and any number of spatial dimensions, maintains sharp triple-junctions without voids or overlaps, can be used for more general velocity fields not near equilibrium, and reduces to the original level set method for two-phase flows. Our method was first applied to domain coarsening in late-stage spinodal decomposition in [12].

The outline of the paper is as follows. In Section 2, we give a brief description of the level set method. In Section 3, we describe the general multi-phase flow problem. In Section 4, we describe the new formulation of the projected level set method. In Section 5, we describe the numerical implementation of this method for incompressible fluid motion. Finally, in Section 6, we present numerical results and give concluding remarks. 


\section{The level set method}

We give here a brief overview of the level set method. While the description of this method will be presented in terms of one-dimensional interfaces in the plane, the analysis readily generalizes to higher dimensions.

Imagine a closed curve $\Gamma$ in the plane propagating normal to itself with speed $F$. We can embed the initial position of the front as the zero level set of a function $\phi$ defined on the plane, the level set function, and then identify the evolution of $\phi$ with the propagation of the front itself through a timedependent initial value problem. In order to derive an equation of motion for this level set function $\phi$, we stipulate that the zero level set of the evolving function $\phi$ always match the propagating interface which means that if $\mathbf{x}(t)$ is a point on the interface, then

$$
\phi(\mathbf{x}(t), t)=0 .
$$

By the chain rule,

$$
\phi_{t}+\nabla \phi(\mathbf{x}(t), t) \cdot \mathbf{x}^{\prime}(t)=0
$$

Since $F$ is the speed in the outward normal direction, then $\mathbf{x}^{\prime}(t) \cdot \mathbf{n}=F$ where $\mathbf{n}$ is the outward normal and can be computed from $\phi$ via $\mathbf{n}=\nabla \phi /\|\nabla \phi\|$. This yields an evolution equation for $\phi$, namely,

$$
\begin{aligned}
& \phi_{t}+F\|\nabla \phi\|=0 \\
& \phi(\mathbf{x}, t=0) \text { given. }
\end{aligned}
$$

This is the level set evolution equation introduced by Osher and Sethian [8].

As analysed by Sethian in [9], the efficient solution of these front propagation problems requires the use of upwind difference schemes borrowed from the solution of hyperbolic conservation laws. A detailed discussion of such schemes in the context of interface propagation can be found in [10].

In order to apply the level set method, the velocity field $F$ itself must be defined on the entire domain of $\phi$, not just the zero level set corresponding to the interface itself. Thus, it is necessary to extend $F$ from the interface into the domain of $\phi$. In [1], a technique was introduced for building this extension velocity field in a highly efficient and accurate manner. It is shown there that if $\phi$ is a signed distance function so that $\|\nabla \phi\|=1$ and the extended velocity field $F$ solves

$$
\nabla F \cdot \nabla \phi=0
$$

then the property $\|\nabla \phi\|=1$ is preserved in all regions where $\phi$ and $F$ are smooth. In other words, this velocity extension preserves the signed distance property of the function $\phi$.

Solving equation (4) can be done efficiently by using the Fast Marching Method [11], which is the optimal technique for solving the Eikonal equation, coupled with a bicubic interpolation scheme for initialization [6]. The Fast Marching Method solves an equation of the form

$$
F\|\nabla \phi\|=1
$$

where $F$ is the interface speed by assigning values to $\phi$ at grid points in an ordered fashion from nearest to farthest from the interface $\phi=0$. This is precisely the same order in which equation (4) must be solved. 
Since the interface $\phi=0$ is defined only implicitly, locating the interface during the course of the evolution is non-trivial. It is necessary in order to initialize the Fast Marching Method described above. In [6], a second-order method for locating the interface given the level set function data on regular grid points, which is based on bicubic interpolation, is proposed. This method for locating the interface is attractive due to its accuracy and its easy extendability to higher dimensions.

\section{Overview of multi-phase interface motion}

We now describe the basics of multi-phase flow and the difficulties it presents for the original level set method. Consider the problem of three phases lying in $\mathbb{R}^{2}$. The original level set method breaks down at triple junctions, points where all three phases meet. It is not possible to build a single smooth function $\phi$ which can simultaneously represent all three interfaces between phases. One obvious choice to correct this is to use three separate level set functions $\phi_{i}$, which are positive in phase $i$ and negative outside.

While the use of multiple level set functions solves the problem of representation, the coupling between the different level set functions requires additional constraints or non-physical results can arise. One such problem occurs in interface flows where the motion of the interface is driven solely by surface tension. Without an additional constraint at the triple junction, the three level set functions will pull away from the triple junction leaving a measurable region in which all three level set functions are negative indicating no phase exists. For more complicated flows, it is also possible, sometimes simply through numerical error, for multiple level set functions to be positive at a point indicating more than one phase at a single point.

Neither of these non-physical situations is acceptable, so attempts to apply the level set method to multi-phase flows have led to modifications, such as those discussed in the introduction, that try to account for these problems. The key to a better scheme for handling multi-phase flows using level set methods is to establish a better mapping between the physical domain and the set of phases, where each point maps onto exactly one of the phases. The resulting mapping must then be coupled to the equations of motion so it is preserved during evolution in time.

\section{Projection method}

In order to describe our representation of the phases, we begin with some notation. Consider the general problem of $n$ phases lying in $\mathbb{R}^{d}$. For each phase $i$, we define $\Omega_{i} \subset \mathbb{R}^{d}$ to be the region occupied by phase $i$. Define $\Gamma_{i}=\partial \Omega_{i}$, the boundary of $\Omega_{i}$, and an $m$-junction as a connected subset of $\Gamma_{i_{1} i_{2} \ldots i_{m}} \equiv \Gamma_{i_{1}} \cap \Gamma_{i_{2}} \cap \cdots \cap \Gamma_{i_{m}}$. Note that, except for degenerate cases, each domain $\Omega_{i}$ has dimension $d$, and each boundary $\Gamma_{i}$ has dimension $d-1$. The interface between phase $i$ and phase $j$, $\Gamma_{i j}=\Gamma_{i} \cap \Gamma_{j}$ also has dimension $d-1$ by virtue of the fact that, for a two-phase system, $\Gamma_{i}=\Gamma_{j}$. However, a triple junction $\Gamma_{i j k}=\Gamma_{i} \cap \Gamma_{j} \cap \Gamma_{k}$ is the intersection of three interfaces, and hence has one lower dimension, $d-2$. In general, an $m$-junction is the intersection of two $(m-1)$-junctions, so the dimension $d_{m}$ of an $m$-junction for $m \geqslant 2$ is given by

$$
d_{m}=d-m+1 \text {. }
$$

Thus, in two dimensions a triple point is the only possible $m$-junction for $m>2$. If four or more phases meet at a junction, we can interpret the junction as the coincidence of multiple triple junctions. In three dimensions, triple lines and quadruple points can exist. In this paper we will focus on triple junctions but the method is applicable to arbitrary values of $m$. 
Triple lines are important in many areas. The wetting of a solid surface by a liquid is controlled by contact line dynamics which give rise to complicated phenomena such as viscous fingering. The rupture of encapsulated droplets and the use of liquid bridges in antifoaming agents are other important processes involving triple lines. Foam calculations also contain a large number of separated regions and thus many high-order junctions. Also in semiconductor manufacturing various processes, such as etching and deposition, produce multiple regions on the underlying structure.

Before describing the projection method we consider the nature of the problem posed by multiple junctions in the level set method. In general we wish any level set function $\phi$ to be a distance function satisfying $\|\nabla \phi\|=1$. If a system contains more than two phases then we define a vector containing the level set function of each phase: $\phi(\mathbf{x})=\left(\phi_{1}(\mathbf{x}), \ldots, \phi_{n}(\mathbf{x})\right) \in \mathbb{R}^{n}$. However there are some constraints on the acceptable values of $\phi(\mathbf{x})$. The errors which may occur at multiple junctions are violations of these constraints. The projection method is a means of evolving the interfaces while satisfying the constraints. The first constraint is simply that at any (off-interface) point $\mathbf{x}$ there must be exactly one positive element of $\phi(\mathbf{x})$, say $\phi_{i}$, meaning that $\mathbf{x}$ lies in $\Omega_{i}$. Because we are using distance functions it follows that $\left|\phi_{i}(\mathbf{x})\right|$ is the shortest distance to $\Gamma_{i}$. It is also true for some $j$ that $\phi_{j}(\mathbf{x})=-\phi_{i}(\mathbf{x})$. In other words this distance is also the shortest distance to $\Gamma_{j}$, the boundary of $\Omega_{j}$. The boundary of any other region, $\Gamma_{k}$, must be an equal or greater distance from $\mathbf{x}$. We now have the following constraints on $\phi(\mathbf{x})$ :

$$
\begin{aligned}
& \phi_{i}(\mathbf{x}) \geqslant 0 \\
& \phi_{j}(\mathbf{x})=-\phi_{i}(\mathbf{x}) \\
& \phi_{k}(\mathbf{x}) \leqslant \phi_{j}(\mathbf{x}), \quad \text { for } k \neq i, j .
\end{aligned}
$$

These are essentially the same conditions as in $[7,14]$. These constraints restrict $\phi(\mathbf{x})$ to an $(n-1)$ dimensional manifold $\Pi$ embedded in $\mathbb{R}^{n}$. An illustration for $n=3$, where $\Pi$ is a three-sided cone, is shown in Fig. 1. This manifold consists of the union of $n(n-1) / 2$ different pieces $\Pi_{i j} \subset \mathbb{R}^{n}$ that are sectors of $(n-1)$-hyperplanes, each labeled by a pair of indices $i, j$ and with $\Pi_{i j} \equiv \Pi_{j i}$. A point $\phi(\mathbf{x}) \in \Pi_{i j}$ if for $i, j, \phi(\mathbf{x})$ satisfies (6)-(8).

There are many well defined maps from $\Pi$ into $\mathbb{R}^{n-1}$, and we will choose one that is piecewise linear, and treats all phases in a symmetric way. To construct this map $\Psi: \Pi \rightarrow \mathbb{R}^{n-1}$, we first introduce some auxiliary maps and spaces.

To properly define Euclidean projections in $\mathbb{R}^{n}$, we use a metric such that the vectors $\mathbf{e}_{i}$ form an orthonormal basis, namely $\mathbf{e}_{i} \cdot \mathbf{e}_{j}=\delta_{i j}$. We can project the space $\mathbb{R}^{n}$ into a hyperplane $H$ that passes through the origin and is normal to a given unitary vector $\mathbf{n}$ given by

$$
P: \mathbb{R}^{n} \rightarrow H, \quad \mathbf{p} \mapsto \mathbf{p}-(\mathbf{n} \cdot \mathbf{p}) \mathbf{n} .
$$

When this map is restricted to $\Pi$ not all choices of $\mathbf{n}$ lead to a one-to-one mapping, but the following symmetric choice does:

$$
\mathbf{n}=(1 / \sqrt{n})(1,1, \ldots, 1)_{e} .
$$

Consider now the projections of the unit vectors $\mathbf{e}_{i}$ into the plane $H$. This gives a set of $n$ vectors $P\left(\mathbf{e}_{i}\right)$, all lying in the plane, and clearly not linearly independent. From this set of vectors, we can construct an orthonormal basis for the $(n-1)$-dimensional space $H$. We apply the GramSchmidt procedure to the set $\left\{P\left(\mathbf{e}_{1}\right), P\left(\mathbf{e}_{2}\right), \ldots\right\}$, in the given order, and arrive at the desired basis 


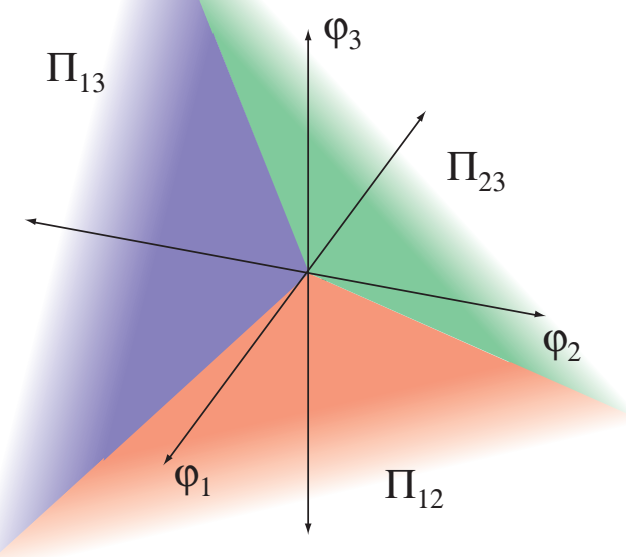

FIG. 1. Illustration of $\Pi$ for $n=3$. Note that the negative $\phi_{3}$ axis lies in $\Pi_{12}$, the negative $\phi_{2}$ axis lies in $\Pi_{13}$, and the negative $\phi_{1}$ axis lies in $\Pi_{23}$.

set: $\left\{\mathbf{h}_{1}, \mathbf{h}_{2}, \ldots\right\}$. Explicitly:

$$
\mathbf{h}_{i}=\frac{1}{\sqrt{(n-i)(n-i+1)}}\left((n-i) \mathbf{e}_{i}-\sum_{j=i+1}^{n} \mathbf{e}_{j}\right) .
$$

We note that the vectors $\mathbf{h}_{i}$ in $\mathbb{R}^{n}$ together with the vector $\mathbf{h}_{n}=\mathbf{n}$ form an orthonormal basis. Therefore we can constuct the projection map $P$ by the $n-1 \times n$ matrix A given by

$$
\mathbf{A}=\left[\begin{array}{c}
h_{1}^{T} \\
\vdots \\
h_{n-1}^{T}
\end{array}\right]
$$

which maps $\mathbb{R}^{n}$ onto $H$ with basis $h_{1}, \ldots, h_{n-1}$ via $\psi=\mathbf{A} \phi$.

The inverse map involves one extra step in order to invert the projection. Given a point $\psi \in H$, it is first mapped back into $\mathbb{R}^{n}$ via the transpose map $\boldsymbol{\theta}=\mathbf{A}^{T} \boldsymbol{\psi}$. Next, we must invert the projection operator. To do this, let $i^{*}, j^{*}$ be indices such that $\theta_{i^{*}} \geqslant \theta_{j^{*}} \geqslant \theta_{k}$ for all $k \neq i^{*}, j^{*}$. Now, the map is inverted by taking

$$
\boldsymbol{\phi}=\boldsymbol{\theta}-\frac{\sqrt{n}}{2}\left(\theta_{i^{*}}+\theta_{j^{*}}\right) \mathbf{n} .
$$


From this construction, we see that

$$
\begin{aligned}
& \phi_{i^{*}}=\theta_{i^{*}}-\frac{\sqrt{n}}{2}\left(\theta_{i^{*}}+\theta_{j^{*}}\right) \frac{1}{\sqrt{n}}=\frac{1}{2}\left(\theta_{i^{*}}-\theta_{j^{*}}\right) \geqslant 0 \\
& \phi_{j^{*}}=\theta_{j^{*}}-\frac{\sqrt{n}}{2}\left(\theta_{i^{*}}+\theta_{j^{*}}\right) \frac{1}{\sqrt{n}}=\frac{1}{2}\left(\theta_{j^{*}}-\theta_{i^{*}}\right)=-\phi_{i^{*}} \\
& \phi_{k}=\theta_{k}-\frac{\sqrt{n}}{2}\left(\theta_{i^{*}}+\theta_{j^{*}}\right) \frac{1}{\sqrt{n}} \leqslant \theta_{j^{*}}-\frac{\sqrt{n}}{2}\left(\theta_{i^{*}}+\theta_{j^{*}}\right) \frac{1}{\sqrt{n}}=\phi_{j^{*}},
\end{aligned}
$$

for all $k \neq i^{*}, j^{*}$. Hence conditions (6)-(8) are satisfied.

For example, when $n=3$, the transformation from $\phi$ to $\psi$ is

$$
\begin{aligned}
& \psi_{1}=\left(2 \phi_{1}-\phi_{2}-\phi_{3}\right) / \sqrt{6} \\
& \psi_{2}=\left(\phi_{2}-\phi_{3}\right) / \sqrt{2}
\end{aligned}
$$

and the inverse transformation is

$$
\begin{aligned}
\theta_{1} & =2 \psi_{1} / \sqrt{6} \\
\theta_{2} & =-\psi_{1} / \sqrt{6}+\psi_{2} / \sqrt{2} \\
\theta_{3} & =-\psi_{1} / \sqrt{6}-\psi_{2} / \sqrt{2} \\
\phi_{1} & =\left(\theta_{1}-\theta_{2}-\theta_{3}+\min \left(\theta_{1}, \theta_{2}, \theta_{3}\right)\right) / 2 \\
\phi_{2} & =\left(-\theta_{1}+\theta_{2}-\theta_{3}+\min \left(\theta_{1}, \theta_{2}, \theta_{3}\right)\right) / 2 \\
\phi_{3} & =\left(-\theta_{1}-\theta_{2}+\theta_{3}+\min \left(\theta_{1}, \theta_{2}, \theta_{3}\right)\right) / 2 .
\end{aligned}
$$

We note that $\psi_{2}$ is equivalent to the dual function $d_{23}=\left(\phi_{2}-\phi_{3}\right) / 2$ (and would be equivalent to other $d_{i j}$ under a rotation of axes). The zero contour of $d_{23}$ (or $\psi_{2}$ ) is the manifold of points equidistant from $\Gamma_{2}$ and $\Gamma_{3}$, containing $\Gamma_{23}$. At a triple junction it bisects the contact angle in $\Omega_{1}$.

\section{Governing equations and numerical implementation}

The class of interfacial motion problems we consider here are those which involve the evolution of $\phi$ according to

$$
\frac{\partial \phi}{\partial t}=\mathbf{f}(\phi) .
$$

A common problem in evolution near $\mathrm{m}$-junctions is the formation of vacuums or overlaps, i.e. grid points where the number of positive $\phi_{i} \mathrm{~s}$ is either zero or greater than one. By using a projected form of (23), these kinds of problems cannot arise. The projected form is obtained by applying the matrix $A$ to both sides of (23) to get

$$
\frac{\partial \boldsymbol{\psi}}{\partial t}=\mathbf{A f}\left(\mathbf{A}^{-1} \psi\right) .
$$

In many instances, such as advection by an external velocity field, (23) has the form $\partial \phi_{i} / \partial t=$ $f\left(\phi_{i}\right)$. In this case (24) can be simplified to $\partial \psi_{i} / \partial t=f\left(\psi_{i}\right)$. 
At grid points adjacent to a junction we solve (24) and then map $\psi^{n+1} \mapsto \phi^{n+1}$ to ensure the proper motion of the junction. Away from the junctions we need only solve (23) for $\phi$. However, if a high-order differencing scheme is used to compute $\mathbf{f}(\phi)$ it is possible that $\left|\mathbf{f}\left(\phi_{i}^{n}\right)\right| \neq\left|\mathbf{f}\left(\phi_{j}^{n}\right)\right|$ near $\Gamma_{i j}$ at points close to a triple junction. This would have the undesired effect of causing $\phi_{i}$ and $\phi_{j}$ to diverge slightly in a region where they are supposed to represent the same interface. To avoid this we evolve the dual function $d_{i j}=\left(\phi_{i}-\phi_{j}\right) / 2$ and update according to $\phi_{i}^{n+1}=d_{i j}^{n+1}$ and $\phi_{j}^{n+1}=-d_{i j}^{n+1}$. We then use the velocity extension method [2] for each $\phi_{i}$ to ensure that it remains a distance function. Thus, we use the following algorithm for the evolution of $\phi$ over a single time step.

\section{ALGORITHM 1}

1. Let $J$ be the set of points that contain a junction in the stencil of the evolution equation.

2. For points in $J$ :

(a) Project $\phi^{n}$ onto $\psi^{n}$.

(b) Update $\psi^{n}$ according to

$$
\psi_{i}^{n+1}=\psi_{i}^{n}+\Delta t f_{i}\left(\psi_{i}^{n}\right)
$$

(c) Project $\psi^{n+1}$ onto $\phi^{n+1}$.

3. For points not in $J$ :

(a) Calculate $\mathbf{f}(\phi)$ (using $d_{i j}$ where a triple junction lies within the stencil of $\mathbf{f}(\phi)$ ).

(b) Calculate the velocity extension for $\mathbf{f}(\phi)$ for each $\phi_{i}$.

(c) Update $\phi^{n}$ according to

$$
\phi_{i}^{n+1}=\phi_{i}^{n}+\Delta t f_{i}\left(\phi_{i}^{n}\right) .
$$

A test problem used in [7] is that of curvature driven interface motion given by

$$
\frac{\partial \phi_{i}}{\partial t}=\left|\nabla \phi_{i}\right| \gamma_{i} \kappa\left(\phi_{i}\right)
$$

where $\kappa$ is the curvature:

$$
\kappa(\phi)=\nabla \cdot \frac{\nabla \phi}{|\nabla \phi|}
$$

We have instead tested our method using the Navier-Stokes equations for a three-phase fluid system which has the advantage of being locally volume conserving. This allows us to compare equilibrium domain shapes with analytical solutions as a means of checking the contact angles. The function $\phi$ is advected by the fluid velocity:

$$
\frac{\partial \phi_{i}}{\partial t}=-\mathbf{u} \cdot \nabla \phi_{i}
$$

Thus we can implement the above algorithm by substituting $\mathbf{f}\left(\phi_{i}\right)=-\mathbf{u} \cdot \nabla \phi_{i}$. The interfaces affect the evolution of the velocity field through the surface tension force which acts to reduce local curvature similar to the former test problem. If we consider the simplified case where viscosity $\mu$, and density $\rho$, are the same in all phases then there are four dimensionless parameters in the equations. These are: the Reynolds number, $R e=\rho u l / \mu$, and a Weber number for each interface, 
$W e_{i j}=\rho l u^{2} / \sigma_{i j}$ where $l$ and $u$ are characteristic length and time scales. The surface tension associated with $\Gamma_{i j}$ is given by $\sigma_{i j}$. The non-dimensional Navier-Stokes equations are:

$$
\begin{gathered}
\frac{\partial \mathbf{u}}{\partial t}=-\nabla p-\mathbf{u} \cdot \nabla \mathbf{u}+r \nabla^{2} \mathbf{u}-\mathbf{F} \\
\nabla \cdot \mathbf{u}=0
\end{gathered}
$$

where $r=1 / \operatorname{Re} . \mathbf{F}$ is the surface tension force. For $n=2$ one can write $\mathbf{F}$ as [3]

$$
\mathbf{F}=w_{12} \kappa\left(\phi_{1}\right) \nabla H\left(\phi_{1}\right)
$$

where $w_{i j}=1 / W e_{i j}$ and $H$ is the Heaviside function. For $n>2$ we decompose the physical surface tensions $w_{i j}$ into the phase specific surface tensions $\tilde{w}_{i}$ such that:

$$
\begin{gathered}
\mathbf{F}=\sum_{1 \leqslant i \leqslant n} \mathbf{F}_{i} \\
\mathbf{F}_{i}=\tilde{w}_{i} \kappa\left(\phi_{i}\right) \nabla H\left(\phi_{i}\right) \\
w_{i j}=\tilde{w}_{i}+\tilde{w}_{j} .
\end{gathered}
$$

We solve (27)-(29) following the methods developed in $[5,13]$ and the algorithm outlined above.

\section{Numerical results}

To ensure that the projection method produces the correct equilibrium contact angles we examined the spreading of a liquid lens (a droplet lying on a free surface). The initial condition is a circular droplet, $\Omega_{3}$, located at a free surface between $\Omega_{1}$ and $\Omega_{2}$. We allow the droplet to relax until a steady shape is reached. The equilibrium contact angles are determined by

$$
\frac{\sin \theta_{1}}{\sigma_{23}}=\frac{\sin \theta_{2}}{\sigma_{13}}=\frac{\sin \theta_{3}}{\sigma_{12}}
$$

and the equilibrium lens shape is the union of two circular sections. The relation between the lens area $A$, its length $d$ (the distance between triple junctions), and the contact angles, $\theta_{i}$, is:

$$
d^{-2}=\frac{1}{8 A}\left(\frac{2\left(\pi-\theta_{1}\right)-\sin \left(2\left(\pi-\theta_{1}\right)\right)}{\sin ^{2}\left(\pi-\theta_{1}\right)}+\frac{2\left(\pi-\theta_{2}\right)-\sin \left(2\left(\pi-\theta_{2}\right)\right)}{\sin ^{2}\left(\pi-\theta_{2}\right)}\right)
$$

Thus, the accuracy of the steady lens shape can be measured by comparing the observed $d$ with the analytical value. This is preferable to measuring the contact angles directly which is made difficult by the curvature of the interfaces.

The length $d$ was measured for a range of surface tensions and the results are listed in Table 1 . The steady droplet shapes for the $128 \times 128$ case are compared with analytical solutions in Fig. 2 . We performed a convergence study for at three different resolutions as shown in Fig. 3. We also 
TABLE 1

Contact angle measurements

\begin{tabular}{llll}
\hline$\left\{\sigma_{i j}\right\}$ & $\theta\left(^{\circ}\right)$ & $d_{\text {exact }}$ & $d_{\text {observed }}$ \\
\hline $1,0.6,0.6$ & 67 & 0.471 & 0.469 \\
$1,0.8,0.8$ & 103 & 0.370 & 0.368 \\
$1,1,1$ & 120 & 0.339 & 0.340 \\
$1,1.2,1.2$ & 131 & 0.319 & 0.320 \\
$1,1.4,1.4$ & 138 & 0.309 & 0.311 \\
\hline
\end{tabular}

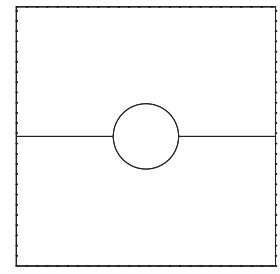

(a)

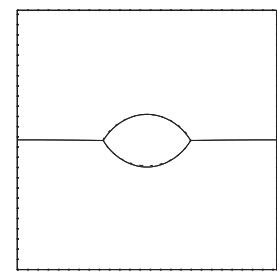

(d)

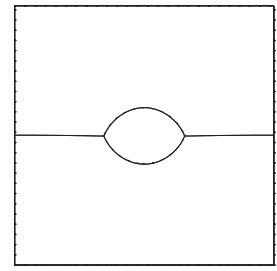

(b)

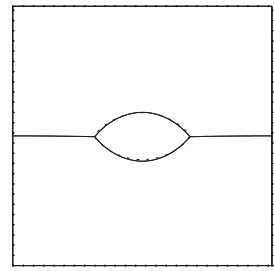

(e)

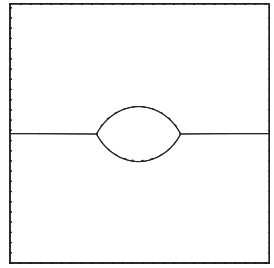

(c)

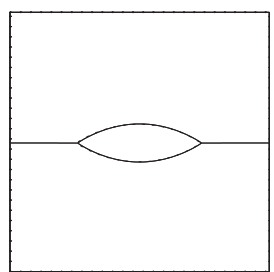

(f)

FIG. 2. Equilibrium droplet shapes. (a) The initial condition. (b)-(f) The steady shapes for $w_{12}=1$ and $w_{13}=w_{23}=$ $1.4,1.2,1,0.8,0.6$ respectively where the upper fluid is phase 1 , the lower fluid is phase 2 , and the droplet is phase 3 . Dashed curves represent the analytical solutions.

examined a case where wetting occurs, $w_{12}=w_{13}=1, w_{23}=10$, to demonstrate that the method handles topological changes appropriately. An evolution sequence is shown in Fig. 4.

To demonstrate an application of our method in a more complex system we show collisions between different phase droplets in three dimensions. The droplets are driven towards each other by a shear flow. We considered two cases: (i) all surface tensions are equal and the droplets stay in contact after colliding and (ii) the droplet-droplet surface tension is too high to maintain a triple line and the droplets slide past each other. Results are presented in Figs 5 and 6. An example from a previous study of late stage spinodal decomposition [12] is shown in Fig. 7. Spinodal decomposition is a phase separation process in which a highly disordered network of bi-(or tri-)continuous domains (see Fig. 7, time $=0.0$ ) is formed by diffusion. In the later stages surface tension induced flow is the driving force for domain growth. 


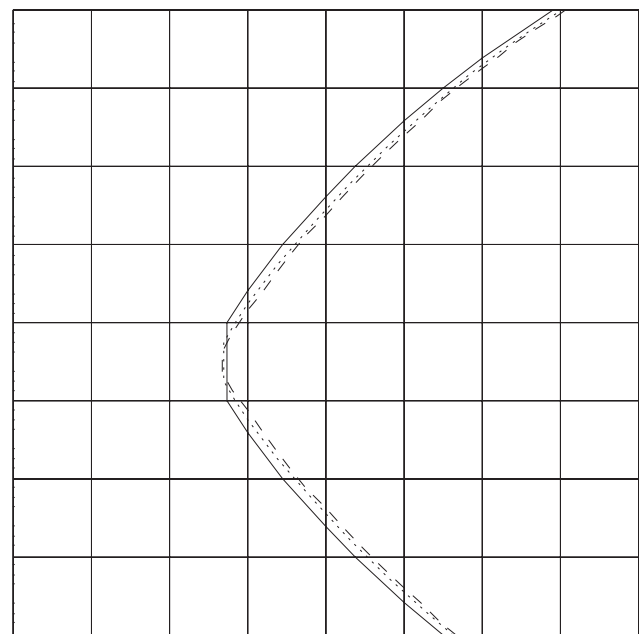

FIG. 3. Convergence study for the case where $w_{13}=w_{23}=1.2$. Grid size is $N \times N$ where $N=64$ for the solid curve, $N=128$ for the dotted curve, and $N=256$ for the dashed curve. Grid lines are for $N=64$.

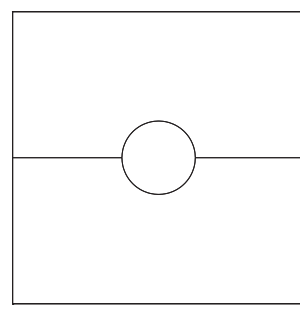

$t=0$

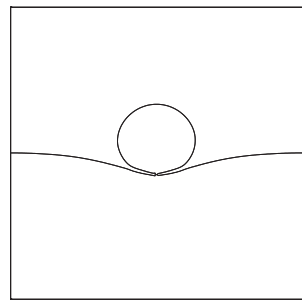

$t=0.15$

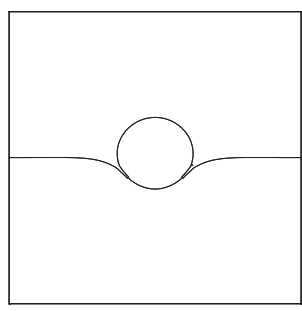

$t=0.05$

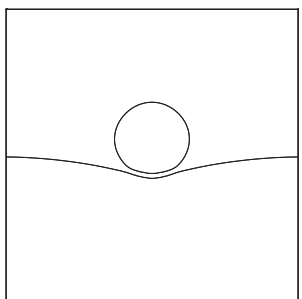

$t=0.3$

FIG. 4. Time sequence of a droplet leaving an interface under surface tension forces. $w_{12}=w_{13}=1, w_{23}=10$. Grid size is $256 \times 256$.

\section{Conclusion}

In summary, we have presented a new algorithm for the modeling of triple junctions using the level set method. This method differs from past efforts in this direction by properly maintaining the correct conditions on the multiple level set functions to ensure that voids and overlaps do not 


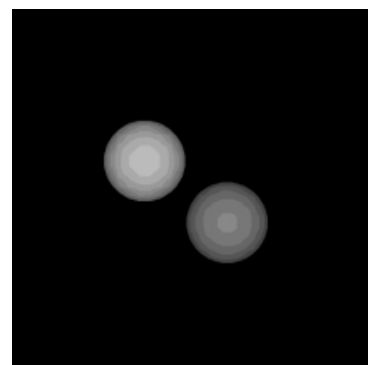

$$
t=0
$$

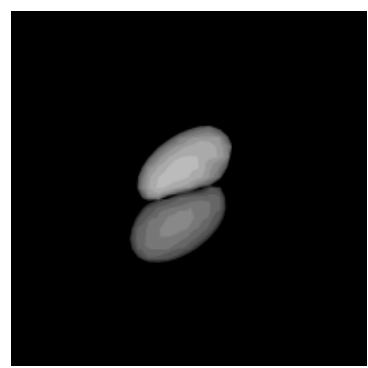

$t=15$

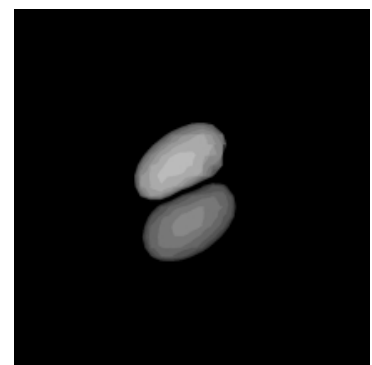

$t=12$

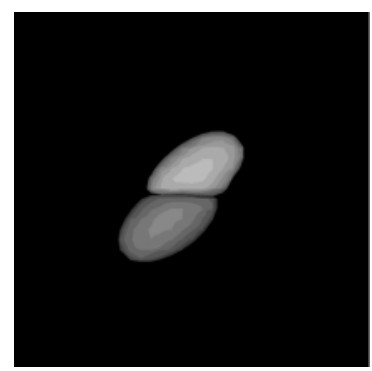

$t=20$

FIG. 5. Time sequence of droplets colliding in 3 D. $w_{12}=w_{13}=w_{23}$ where the droplets are phases 2 and 3 and the external fluid is phase 1 . The grid size is $32 \times 32 \times 32$.

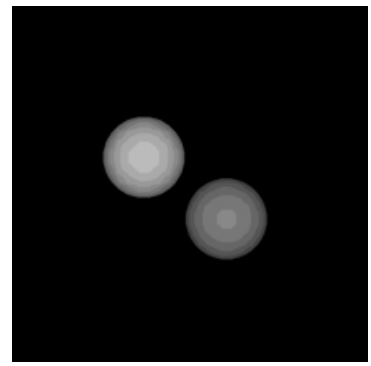

$t=0$

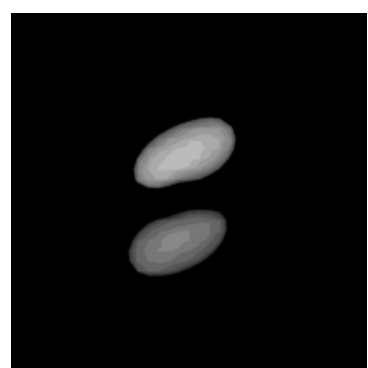

$t=15$

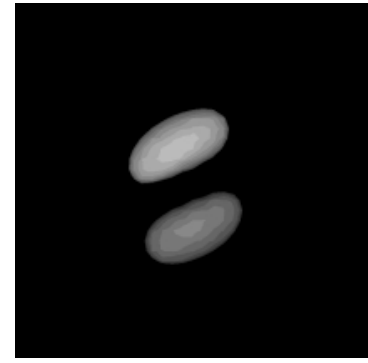

$t=12$

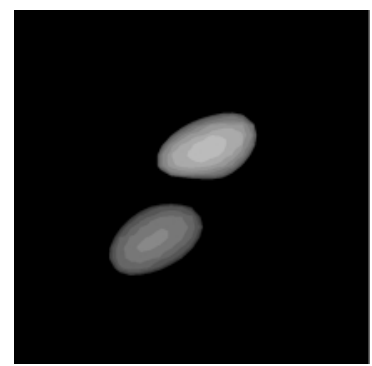

$t=20$

FIG. 6. Time sequence of droplets passing in three dimensions. $w_{23}=1, w_{12}=w_{13}=0.1$. 


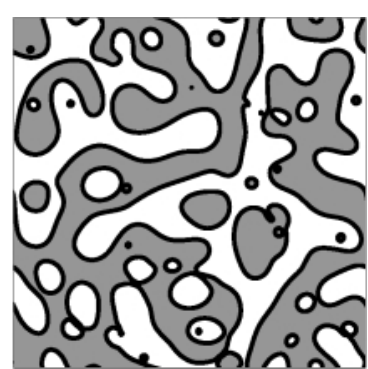

time $=0.0$

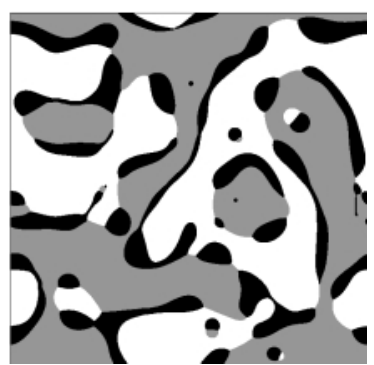

time $=0.023$

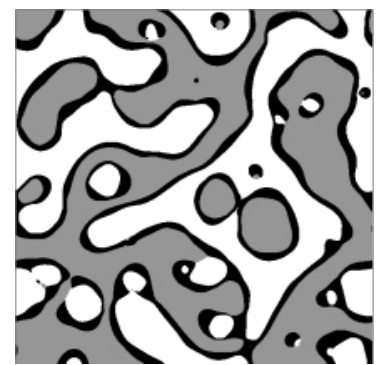

time $=0.008$

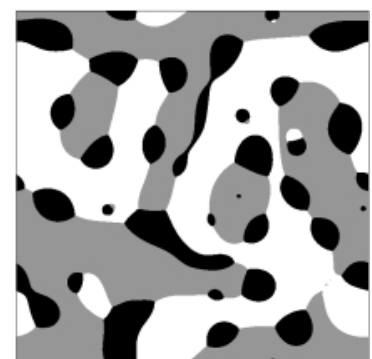

time $=0.039$

FIG. 7. Domain growth in late-stage spinodal decomposition for a three phase system. $w_{12}=w_{13}=w_{23}$. Boundary conditions are periodic. Reprinted from [12].

occur, while at the same time decoupling the level set representation from the underlying velocity field. The result is an algorithm that eliminates overlaps and voids, and can be used for dynamic simulations (not just relaxation to equilibrium). In regions where only two phases are present, the method reduces to the original level set method which has been proven successful in many areas. Finally, we have demonstrated the effectiveness of this method for solving complex multi-phase flow.

\section{Acknowledgements}

David Chopp was supported in part by the NSF/DARPA VIP program under award 96-15877. Kurt Smith was supported by a National Science Foundation IGERT grant titled 'Dynamics of Complex Systems in Science and Engineering'.

\section{REFERENCES}

1. Adalsteinsson, D. \& Sethian, J. A. The fast construction of extension velocities in level set methods. J. Comp. Phys. 148, (1999) 2-22.

2. Adalsteinsson, D. \& Sethian, J. A. The fast construction of extension velocities in level set methods. J. Comp. Phys. 148, (1999) 2-22.

3. BRACKBill, J. U., Kothe, D. B., \& ZEMACH, C. A continuum method for modeling surface tension. J. Comp. Phys. 100, (1992) 335-354. 
4. Chan, T. F. \& Vese, L. A. Active contours without edges. IEEE Trans. Image Proc. 10, (2001) 266277.

5. Chang, Y. C., Hou, T. Y., Merriman, B., \& Osher, S. A level set formulation of eulerian interface capturing methods for incompressible fluid flows. J. Comp. Phys. 124, (1996) 449-464.

6. CHOPP, D. L. Some improvements of the fast marching method. SIAM J. Sci. Comp. 23, (2001) 230-244.

7. Merriman, B., Bence, J. K., \& Osher, S. J. Motion of multiple junctions- a level set approach. $J$. Comp. Phys. 112, (1994) 334-363.

8. Osher, S. \& Sethian, J. A. Fronts propagating with curvature dependent speed: algorithms based on Hamilton-Jacobi formulation. J. Comp. Phys. 79, (1988) 12-49.

9. Sethian, J. A. Numerical methods for propagating fronts. In: ConCUS, P. \& FInN, R. (eds), Variational Methods for Free Surface Interfaces. pp. 155-164. Springer, New York (1987).

10. Sethian, J. A. Level Set Methods: Evolving Interfaces in Geometry, Fluid Mechanics, Computer Vision and Material Science. Cambridge University Press, Cambridge (1996).

11. Sethinan, J. A. A marching level set method for monotonically advancing fronts. Proceedings of the National Academy of Sciences. pp. 1591-1595. 93, (1996).

12. Smith, K. A., Solis, F. J., TaO, L., Thornton, K., \& Olvera de la Cruz, M. Domain growth in ternary fluids: a level set approach. Phys. Rev. Lett. 84, (2000) 91-94.

13. Sussman, M., SMereka, P., \& OSher, S. A level set approach for computing solutions to incompressible 2-phase flow. J. Comp. Phys. 114, (1994) 146-159.

14. Zhao, H.-K., Chan, T., Merriman, B., \& Osher, S. A variational level set approach to multiphase motion. J. Comp. Phys. 127, (1996) 179-195.

15. Zhao, H-K, Merriman, B., Osher, S., \& Wang, L. Capturing the behavior of bubbles and drops using the variational level set approach. J. Comp. Phys. 143, (1998) 495-518. 